\title{
Willingness, beliefs, and barriers regarding the COVID-19 vaccine in Saudi Arabia: a multiregional cross-sectional study
}

\author{
Noura Altulahi', Shouq AlNujaim², Azzam Alabdulqader ${ }^{3}$, Abdullah Alkharashi $^{3}$, Assaf AlMalki ${ }^{3}$, Faisal AlSiari ${ }^{3}$, \\ Yara Bashawri ${ }^{4}$, Sarah Alsubaie ${ }^{5}$, Dayel AlShahrani ${ }^{6}$ and Yara AlGoraini ${ }^{7^{*}}$
}

\begin{abstract}
Background: The coronavirus disease 2019 (COVID-19) has spread worldwide, and the vaccine remains the ultimate cornerstone to overcoming its long-term impact. Vaccine hesitancy might obstruct the effort to achieve herd immunity and eradicate the virus. We assessed Saudi Arabian individuals' willingness, beliefs, and barriers regarding the COVID-19 vaccine and their adherence to preventive measures during and after the pandemic.

Methods: A self-administered electronic validated questionnaire was distributed among the five major regions in Saudi Arabia between November and December 2020. The questionnaire addressed the sociodemographic data, beliefs, potential barriers, parents' acceptance of COVID-19 vaccination for their children, and adherence to protective measures during and after the pandemic.

Results: Of 8,056 participants, 4,218 (52.4\%) of a non-representative sample were willing to be vaccinated against COVID-19. Being a young adult, male, having less than a high school degree, being a smoker, having a chronic disease, and having a history of seasonal influenza vaccine uptake were positive predictors of COVID-19 vaccine acceptance. Hesitant participants reported concerns about vaccine side effects and safety as the main barriers to accepting the COVID-19 vaccine. Some refusers (26.1\%) declared that they would reconsider vaccination only if the safety and effectiveness of the vaccine were reported by more studies.

Conclusions: Our study revealed a promising willingness to accept the vaccine among the population, with positive beliefs and attitudes toward COVID-19 vaccination. However, a considerable proportion of the population was reluctant to accept the vaccine. Thus, publicly providing information about vaccine safety and implementing health education programs is crucial for increasing the public's confidence in the vaccine.
\end{abstract}

Keywords: Willingness COVID-19 vaccine, Vaccination, Beliefs, Barriers, Saudi Arabia

\section{Background}

The coronavirus disease 2019 (COVID-19), caused by the severe acute respiratory syndrome coronavirus 2, has negatively impacted the global health capacity and

${ }^{*}$ Correspondence: Yalgoraini@Kfmc.Med.Sa

${ }^{7}$ Pediatric Emergency Department, King Fahad Medical City, Riyadh, Saudi Arabia

Full list of author information is available at the end of the article economy [1]. About $80 \%$ of infected people recover without requiring hospitalization; however, one out of every five people with COVID-19 becomes critically ill [2]. Saudi Arabia (SA) has been affected by a few pandemics, including the Middle East respiratory syndrome coronavirus and the ongoing COVID-19 [3]. The COVID-19 infection quickly spread to SA, causing the deaths of thousands. According to World Health Organization report between January 3, 2020 and November 17, 2021, 
there have been 549,297 confirmed cases of COVID-19 with 8,818 reported deaths [4]. SA public health authorities have intervened by restricting the population in protective measures such as maintaining social distance, wearing masks, and using hand sanitizers [5]. Despite using many drugs and protective measures to prevent and treat this disease, the global demand for a vaccine is inevitable [6]. The government's current focus is to introduce vaccines to the public to reach sufficient global immunity; however, no vaccines had been approved by the time of conducting this study [7].

Vaccination is one of the most effective ways to prevent the spread of disease and reduce complications associated with it. Vaccine acceptance and maintenance of high equitable vaccination uptake among all populations is not straightforward [8]. Several international and a few local studies have examined the willingness to accept the vaccine and the beliefs and barriers associated with it [9-20]. In Italy, a study reported a reduction in the willingness to receive the COVID-19 vaccine [9]. In addition, a study in the US found that $20 \%$ of the participants would refuse the vaccine [10]. Moreover, studies in the US and the UK found that adults showed a lower willingness and acceptance of the vaccination for their children than for themselves [11]. In contrast, a study in France showed that most participants were willing to receive the vaccine against COVID-19, similar to the report of a European study $[12,13]$. A global study surveyed the potential acceptance of the COVID-19 vaccine in 19 different countries. Of the total population, $71.5 \%$ stated their willingness to accept the vaccine. However, differences in acceptance rates fluctuated from almost 90\% (in China) to less than 55\% (in Russia) [14].

Several factors may be associated with a higher probability of vaccine acceptance among a population, as reported by another US study, such as increasing the efficacy and decreasing the incidence of considerable adverse effects of the vaccine [15]. In China, most participants believed that doctors' recommendations are an important factor in vaccine acceptance (80.6\%), similar to another US study $[16,17]$. Another study in China reported that healthcare workers (HCWs) showed a higher willingness for vaccine acceptance than the general population [18]. In SA, a study showed that only $44.7 \%$ of the participants planned to receive the vaccine, $78.9 \%$ had positive beliefs, and $79.9 \%$ were concerned about its side effects [19]. Another study in SA showed that most participants were interested in receiving vaccination [20].

The COVID-19 pandemic has spread worldwide, and the vaccine remains the key to ending its negative impact. This study aimed to assess the willingness, beliefs, and barriers regarding COVID-19 vaccination among SA's population. Our findings play a role in advancing how the population's beliefs and attitudes toward vaccination could change during an unusual public health crisis; this requires designing effective behavior change communication campaigns by the healthcare system such as conducting campaigns at malls, mosques, and schools, as well as in the media, to increase awareness among the public. Currently, the number of Saudi and international studies on COVID-19 vaccine hesitancy are substantial; however, our results will further enlighten the healthcare professionals and policymakers to address people's beliefs and concerns regarding vaccinations. We also provide results on parents' attitudes toward their own as well as their child's vaccination, which remains an active research area. The importance of our results is not confined only to the current scenario and can be used to help combat any possible pandemics in the future. Such data will also help educate the public regarding vaccination hesitancy and acceptance by means tailored based on the public's beliefs and concerns.

\section{Methods \\ Study design}

This was a cross-sectional study. A self-administered electronic questionnaire was distributed between November and December 2020 through social networking sites, such as WhatsApp and Twitter, targeting the Saudi population. We decided to use the self-administered electronic questionnaire to reach the population as The Saudi General Authority for Statistics reported that $88.6 \%$ of the Saudi population from the age of 15 years and older spend time on the Internet; among them, 97.34\% spend their internet time on social media [21]. Prior screening questions were provided to ensure that the participants met the inclusion criteria, including age ( $\geq 18$ years) and living in SA. The study was approved by the Institutional Review Board of King Fahad Medical City (no. IRB00010471).

\section{Questionnaire development}

We conducted a literature review to assess and extract any related questions used in previous studies regarding the COVID-19 vaccine [14, 16-20, 22-25]. The first step of validation was face validation, which was conducted by three independent field experts. They reviewed the survey questions to capture the study objectives and ensure that they were not misleading, vague, or double-barreled. The second step was pilot testing among the population to minimize ambiguity and enhance clarity. Finally, the questionnaire was distributed in Arabic and English, and the translation was performed using a backward-forward design. The survey addressed five main sections: sociodemographic variables, participants' beliefs about the 
COVID-19 vaccination, their willingness to get the vaccine, any potential barriers to the vaccination, parents' willingness for their children to get the vaccine, and participants' behavior during and after the pandemic.

\section{Sample size justification}

While presuming that $50 \%$ of the Saudi adult population have an awareness of the COVID-19 pandemic and allowing $5 \%$ error on either side of the stated prevalence (or $90 \%$ power) at a $95 \%$ confidence interval (CI), the estimated sample size was derived using PASS 11 software by Cochran's method. The estimated sample size for each of the geographical regions was 1,574 .

Therefore, a population of 7,870 participants were derived by multiplying the initial target sample size by five to reflect the five geographic areas.

Our collected sample was non-representative of the Saudi population; it deviates from the national population census from the SA General Authority for Statistics [26] by age group and sex. Most of our sample were women $(54.2 \%)$, while $57.7 \%$ of SA's citizens are men. Moreover, most participants were young adults (43.8\%), followed by middle-aged adults (43.2\%), and older adults $(13.0 \%)$; in contrast, the proportions in SA are 14.5\%, $52 \%$, and $8.9 \%$, respectively. No other sociodemographic information such as nationality, residence, marital status, educational level, or professional background were available.

\section{Data management and analysis}

Demographic and clinical characteristics of the study participants were reported as counts (percentage). Chisquare test was used to assess the signifcance of the association (contingency) between COVID-19 vaccine acceptance and sociodemographic variables. Factors that were potential predictors of willingness were analyzed using logistic regression analyses. Stepwise multivariate logistic regression analyses were used with factors that were significant $(\leq 0.30)$ in the univariate analysis. All analyses were performed using SPSS version 25.0 (SPSS Inc., Chicago, IL, USA). A p-value (two-tailed) $\leq 0.05$ was considered significant.

\section{Results}

In total, 8,056 participants completed the survey (Table 1), and they were mostly young (18-28 years; $43.8 \%$ ) or middle-aged ( $29-50$ years; $43.2 \%$ ) adults. More than half of the participants were women $(54.2 \%)$, and most had at least a university degree (75.8\%). A minority were healthcare providers (15.2\%), and more than two-thirds (83\%) denied having any chronic diseases. Most participants did not have any allergies (82.9\%), and a small proportion of them were smokers (19.3\%).
Approximately half of the participants had received a seasonal influenza vaccine before (51.1\%), and only a few reported that they had tested positive for COVID-19 (11.7\%).

Of the 8,056 participants, $47.6 \%$ were not willing to receive the vaccine, while $52.4 \%$ were willing to be vaccinated against COVID-19. The proportion of those who were willing to accept the vaccine was higher among the young adults $(56.4 \%)$, men (55\%), those with chronic diseases (55.5\%), smokers (58.4\%), those with a high school degree (56.8\%), those who had received seasonal influenza vaccine $(57 \%)$, and those with less than a high school degree (57\%; Table 1).

A logistic regression analysis of the positive predictors of COVID-19 vaccine acceptance among the participants showed that, compared to their respective counterparts, young adults (odds ratios: 1.781 [95\% CI: 1.528-2.075]), male participants (odds ratio: 1.153 [95\% CI: 1.047$1.269]$ ), those who had received seasonal influenza vaccine (odds ratio: 1.508 [95\% CI: 1.378-1.649]), those with comorbidities (odds ratio: 1.285 [95\% CI: 1.131-1.460]), smokers (odds ratio: 1.277 [95\% CI: 1.133-1.439]), those with less than a high school degree (odds ratio: 1.339 [95\% CI: 1.003-1.787]), and those with a high school degree (odds ratio: 1.247 [95\% CI: 1.117-1.393]) were more willing to receive the COVID-19 vaccine (Table 2).

Most participants had positive beliefs regarding the safety $(65.4 \%)$ and effectiveness $(67.2 \%)$ of the vaccine (Table 3). Additionally, they agreed on the importance of the vaccine in eradicating COVID-19. However, approximately half of the participants feared contracting COVID-19 (49.6\%), and some considered themselves at high risk of contracting the disease (23.3\%). Moreover, more than half of the participants believed that they might contract COVID-19 even after being immunized (51.9\%). Nonetheless, more than half were willing to receive the COVID-19 vaccine when available (52.4\%; Table 3).

Table 4 shows the barriers associated with COVID19 vaccine acceptance. Many vaccine refusers were concerned about the side effects (60.03\%) and safety (40.36\%). Moreover, some participants believed that developing immunity following the COVID-19 infection is better than vaccination (28.06\%). Others $(27.85 \%)$ believed that the vaccine was unnecessary because they were practicing protective measures such as frequent hand washing and wearing masks. Approximately $24.47 \%$ did not believe that the vaccine would stop the infection, and $15.37 \%$ believed that the vaccination is a conspiracy. Vaccine refusers declared that they would reconsider if the safety and effectiveness were reported by more studies $(54.85 \%)$, it was mandated by the government $(40.18 \%)$, their physicians 
Table 1 Sociodemographic and Predicting factors of COVID-19 vaccine acceptance $n=8056$

\begin{tabular}{|c|c|c|c|c|}
\hline \multirow[t]{3}{*}{ Variables } & \multirow{3}{*}{$\begin{array}{l}\text { Total Population } n=8056 \\
(\%)\end{array}$} & \multicolumn{2}{|c|}{ If COVID-19 vaccination is available, I will take it } & \multirow[b]{3}{*}{$P$-value } \\
\hline & & NO (\%) & YES (\%) & \\
\hline & & $n=3838(47.6 \%)$ & $n=4218(52.4 \%)$ & \\
\hline \multicolumn{5}{|l|}{ Age } \\
\hline Young Adults (18-28 years) & $3526(43.8 \%)$ & $1539(43.6 \%)$ & $1987(56.4 \%)$ & \multirow[t]{3}{*}{$<0.0001^{* * * *}$} \\
\hline Middle aged adults (29-50 years) & $3482(43.2 \%)$ & $1744(50.1 \%)$ & $1738(49.9 \%)$ & \\
\hline Older adults (older than 50 years) & $1048(13.0 \%)$ & $555(53 \%)$ & $493(47 \%)$ & \\
\hline \multicolumn{5}{|l|}{ Sex } \\
\hline Male & $3688(45.8 \%)$ & $1658(45 \%)$ & $2030(55 \%)$ & \multirow[t]{2}{*}{$<0.0001^{* * * *}$} \\
\hline Female & $4368(54.2 \%)$ & $2180(49.9 \%)$ & $2188(50.1 \%)$ & \\
\hline \multicolumn{5}{|l|}{ Nationality } \\
\hline Saudi & $7483(92.9 \%)$ & $3577(47.8 \%)$ & $3906(52.2 \%)$ & \multirow[t]{2}{*}{0.298} \\
\hline Non-Saudi & $573(7.1 \%)$ & $261(45.5 \%)$ & $312(54.5 \%)$ & \\
\hline \multicolumn{5}{|l|}{ Residence } \\
\hline Middle region & $2893(35.9 \%)$ & $1422(49.2 \%)$ & $1471(50.8 \%)$ & \multirow[t]{5}{*}{$<0.0001^{* * * *}$} \\
\hline East region & $2129(26.4 \%)$ & $1076(50.5 \%)$ & $1053(49.5 \%)$ & \\
\hline Western region & $1997(24.8 \%)$ & $912(45.7 \%)$ & $1085(54.3 \%)$ & \\
\hline North region & $327(4.1 \%)$ & $146(44.6 \%)$ & 181 (55.4\%) & \\
\hline South region & $710(8.8 \%)$ & $282(39.7 \%)$ & $428(60.3 \%)$ & \\
\hline \multicolumn{5}{|l|}{ Marital status } \\
\hline Single & $3511(43.6 \%)$ & $1561(44.5 \%)$ & $1950(55.5 \%)$ & \multirow[t]{4}{*}{$<0.0001^{* * * *}$} \\
\hline Married & $4266(53.0 \%)$ & $2128(49.9 \%)$ & $2138(50.1 \%)$ & \\
\hline Widowed & $40(0.5 \%)$ & $22(55 \%)$ & $18(45 \%)$ & \\
\hline Divorced/separated & $239(3.0 \%)$ & $127(53.1 \%)$ & 112 (46.9\%) & \\
\hline \multicolumn{5}{|l|}{ Education } \\
\hline Less than High school & $200(2.5 \%)$ & $86(43 \%)$ & $114(57 \%)$ & \multirow[t]{3}{*}{$<0.0001^{* * * *}$} \\
\hline High school & $1747(21.7 \%)$ & $754(43.2 \%)$ & $993(56.8 \%)$ & \\
\hline University and higher & $6109(75.8 \%)$ & $2998(49.1 \%)$ & $3111(50.9 \%)$ & \\
\hline \multicolumn{5}{|l|}{ Professional background } \\
\hline Healthcare provider & $1228(15.2 \%)$ & $599(48.8 \%)$ & $629(51.2 \%)$ & \multirow[t]{3}{*}{0.189} \\
\hline Employed & $4294(53.3 \%)$ & $2069(48.2 \%)$ & $2225(51.8 \%)$ & \\
\hline Unemployed & $2534(31.5 \%)$ & $1170(46.2 \%)$ & $1364(53.8 \%)$ & \\
\hline \multicolumn{5}{|l|}{ Do you have any chronic disease? } \\
\hline No & $6683(83.0 \%)$ & $3227(48.3 \%)$ & $3456(51.7 \%)$ & \multirow[t]{2}{*}{$0.011^{* *}$} \\
\hline Yes & $1373(17.0 \%)$ & $611(44.5 \%)$ & $762(55.5 \%)$ & \\
\hline \multicolumn{5}{|l|}{ Do you have any allergy? } \\
\hline No & $6682(82.9 \%)$ & $3149(47.1 \%)$ & $3533(52.9 \%)$ & \multirow[t]{2}{*}{$0.041^{*}$} \\
\hline Yes & $1374(17.1 \%)$ & $689(50.1 \%)$ & 685(49.8\%) & \\
\hline Are you a smoker? & & & & \\
\hline No & $6499(80.7 \%)$ & $3190(49.1 \%)$ & $3309(50.9 \%)$ & $<0.0001^{* * * *}$ \\
\hline Yes & $1557(19.3 \%)$ & $648(41.6 \%)$ & 909 (58.4\%) & \\
\hline Did you get the seasonal influenza $v$ & & & & \\
\hline No & $3941(48.9 \%)$ & $2070(52.5 \%)$ & $1871(47.5 \%)$ & $<0.0001^{* * * *}$ \\
\hline Yes & $4115(51.1 \%)$ & $1768(43 \%)$ & $2347(57 \%)$ & \\
\hline Have you been tested positive for $\mathrm{CC}$ & & & & \\
\hline No & $7117(88.3 \%)$ & $3422(48.1 \%)$ & $3695(51.9 \%)$ & $0.029^{*}$ \\
\hline Yes & $939(11.7 \%)$ & $416(44.3 \%)$ & $523(55.7 \%)$ & \\
\hline If yes, were you hospitalized for COV & & & & \\
\hline No & $878(93.5 \%)$ & $398(45.3 \%)$ & $480(54.7 \%)$ & $0.018^{*}$ \\
\hline Yes & $61(6.5 \%)$ & $18(29.5 \%)$ & $43(70.5 \%)$ & \\
\hline
\end{tabular}


Table 1 (continued)

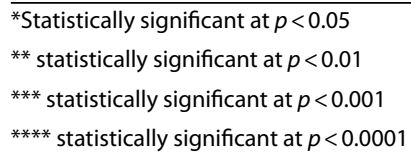

Table 2 Logistic regression analysis of positive predicting factors of COVID-19 vaccine acceptance

\begin{tabular}{|c|c|c|c|c|}
\hline \multirow[b]{2}{*}{ Variables } & \multirow[b]{2}{*}{ P-value } & \multirow[b]{2}{*}{ OR } & \multicolumn{2}{|c|}{ 95\% C.I.for EXP(B) } \\
\hline & & & Lower & Upper \\
\hline Sex Male: Female & $0.004^{* *}$ & 1.153 & 1.047 & 1.269 \\
\hline Comorbidity Yes: No & $<0.0001^{* * *}$ & 1.285 & 1.131 & 1.460 \\
\hline Allergy Yes: No & $0.020^{*}$ & 0.868 & 0.771 & 0.978 \\
\hline Smoking Yes: No & $<0.0001^{* * * *}$ & 1.277 & 1.133 & 1.439 \\
\hline Flu vaccine Yes: No & $<0.0001^{* * *}$ & 1.508 & 1.378 & 1.649 \\
\hline Age & $<0.0001^{* * *}$ & & & \\
\hline Young aged adult: Older adults & $<0.0001^{* * *}$ & 1.781 & 1.528 & 2.075 \\
\hline Middle aged adults: Older aged adults & $0.001^{* * *}$ & 1.291 & 1.112 & 1.499 \\
\hline Education & $<0.0001^{* * * *}$ & & & \\
\hline Less than High school: University and Higher & $0.048^{*}$ & 1.339 & 1.003 & 1.787 \\
\hline High school: University and Higher & $<0.0001^{* * *}$ & 1.247 & 1.117 & 1.393 \\
\hline
\end{tabular}

*Statistically significant at $p<0.05$

** statistically significant at $p<0.01$

*** statistically significant at $p<0.001$

**** statistically significant at $p<0.0001$

recommended it to them $(22.49 \%)$, and it was required to protect their family $(22.02 \%)$. However, some still refused regardless of the situation (17.61\%).

Of the 8,056 participants, $42 \%$ had children aged $<18$ years. Half of the parents were willing to accept vaccination for their children (51.67\%). The most common reason for refusing was their lack of belief in the efficacy of the vaccine (65.44\%), followed by believing that developing immunity following COVID-19 infection was better than getting the vaccine (34.07\%) and concerns about the side effects (31.43\%).

Most participants generally showed good compliance regarding the use of face masks (90.4\%), hand washing or sanitization $(82.7 \%)$, avoiding crowded places (76.1\%), and maintaining social distance $(72.7 \%)$ during the pandemic $(98.4 \%)$; they were planning to continue these measures even after the pandemic (89\%). Only $2.6 \%$ of the participants reported that they did not use any protective measures during the pandemic. Overall, $41.7 \%$ of the participants reported that they would stop wearing face masks, $77.3 \%$ would reduce regular hand washing/sanitization, $55.3 \%$ would stop avoiding crowded places, and $29.8 \%$ would stop maintaining social distance after the pandemic. Only $12.2 \%$ reported that they would not use any protective measures after the pandemic.

\section{Discussion}

The Kingdom of SA has taken drastic measures to mitigate the burden of the COVID-19 pandemic substantially. Despite these tremendous efforts, the vaccine remains the key to ending the long-term impact of COVID-19 [27]. The acceptance and refusal of the vaccination generally vary both locally and internationally, even among individuals of the same sex, age, and class [28]. We assessed the willingness, beliefs, and barriers regarding the COVID-19 vaccination across the five central regions in SA. This highlights the degree of confidence and willingness among the population, thus elucidating the factors influencing people's decisions to accept an emergency-use released vaccine in helping authorities better manage the pandemic.

Women's statistical dominance has also been reported in similar international and local studies [14-20, 22-25]. Our results regarding the predominance of younger adults and university degree holders in vaccine acceptance are consistent with those of other studies [14-16, $18,19,24,25,28]$. Furthermore, most participants (83\%) did not have any chronic illnesses, which is consistent with studies from other Arab countries and France [19, 22, 29].

These consistencies could be because of biological, social, technological, cultural, and economic factors 
Table 3 Participants' beliefs toward COVID-19 vaccination $n=8056$

\begin{tabular}{|c|c|c|}
\hline Variables & & Count (\%) \\
\hline \multirow[t]{2}{*}{ I fear catching a COVID-19 infection } & No & $\begin{array}{l}4061 \\
(50.4 \%)\end{array}$ \\
\hline & Yes & $\begin{array}{l}3995 \\
(49.6 \%)\end{array}$ \\
\hline \multirow[t]{2}{*}{ I think I am at high risk of catching a COVID-19 infection } & No & $\begin{array}{l}6175 \\
(76.7 \%)\end{array}$ \\
\hline & Yes & $\begin{array}{l}1881 \\
(23.3 \%)\end{array}$ \\
\hline \multirow[t]{2}{*}{ I think COVID-19 vaccine is important } & No & $\begin{array}{l}2151 \\
(26.7 \%)\end{array}$ \\
\hline & Yes & $\begin{array}{l}5905 \\
(73.3 \%)\end{array}$ \\
\hline \multirow[t]{2}{*}{ I think COVID-19 vaccine, whenever available, would be safe } & No & $\begin{array}{l}2785 \\
(34.6 \%)\end{array}$ \\
\hline & Yes & $\begin{array}{l}5271 \\
(65.4 \%)\end{array}$ \\
\hline \multirow[t]{2}{*}{ I think COVID-19 vaccine, whenever available, would be effective } & No & $\begin{array}{l}2639 \\
(32.8 \%)\end{array}$ \\
\hline & Yes & $\begin{array}{l}5417 \\
(67.2 \%)\end{array}$ \\
\hline \multirow[t]{2}{*}{ I think I might get infected with COVID-19 after immunization } & No & $\begin{array}{l}3871 \\
(48.1 \%)\end{array}$ \\
\hline & Yes & $\begin{array}{l}4185 \\
(51.9 \%)\end{array}$ \\
\hline \multirow[t]{2}{*}{ I think the best way to avoid COVID-19 infection is by getting the vaccine } & No & $\begin{array}{l}3024 \\
(37.5 \%)\end{array}$ \\
\hline & Yes & $\begin{array}{l}5032 \\
(62.5 \%)\end{array}$ \\
\hline \multirow[t]{2}{*}{ If COVID-19 vaccination is available, I will take it } & No & $\begin{array}{l}3838 \\
(47.6 \%)\end{array}$ \\
\hline & Yes & $\begin{array}{l}4218 \\
(52.4 \%)\end{array}$ \\
\hline
\end{tabular}

[14-20, 22-25]. At the time of this study, we did not refer to any local or international studies where they consider the history of allergy, hospitalization, or smoking as an element of their sociodemographic. However, we believed that it would be beneficial for investigating factors associated vaccine acceptance and thus included these factors.

The rates of acceptance and hesitancy toward any vaccine differ worldwide [20]. This variation may be influenced by location, time, and contextual human behavior $[8,24,30]$. Our study demonstrated that half of the participants declared their willingness to get vaccinated (52.4\%). It was reassuring that most participants were not vaccine-hesitant, consistent with the findings of other studies in the US (67\%) [17], France (77.6\%)]12[, UK (64\%) [22], China (91.3\%) [16], Europe (73.9\%) [13[, SA (64.72\%) [20[, and Egypt (73\%) [23]. In studies from Jordan, Kuwait, and other Arab countries, the acceptance rate for COVID-19 vaccines was only $29.4 \%$ [24]. Additionally, a survey conducted in SA among 3,101 participants showed that $55.3 \%$ were hesitant to accept the COVID-19 vaccine [19]. However, a participant's willingness to accept the vaccine does not necessarily assure their actual future uptake [14].

Few studies have shown that willingness to receive a vaccine is linked to several predictive factors and barriers, including distrust in the research; the safety, efficacy, and adverse outcomes of the vaccine; risk of being infected with the virus; a lack of knowledge about the nature of vaccine-preventable diseases; and misconceptions and misinformation related to the vaccine [9-11, 13-20, 22-25]. Our study highlighted several factors that may increase or decrease people's willingness to receive the vaccine. Concerns over vaccine side effects was the most common barrier to vaccine acceptance among refusers, consistent with the reports from SA [19], the US [12], China [18], and Europe [13]. Another main factor and barrier found in this study was the lack of trust regarding the safety of the vaccination, which was similarly reported in previous studies from Italy, the US, and 
Table 4 Participants' barriers associated with acceptance of COVID-19 vaccination $n=3838$

\begin{tabular}{|c|c|}
\hline Barriers & $\begin{array}{l}\text { Vaccine refusers } \\
n=3838(47.6 \%) \\
\text { Count }(\%)\end{array}$ \\
\hline I am concerned about the vaccine's side effects & $\begin{array}{l}2304 \\
(60.03 \%)\end{array}$ \\
\hline I don't believe that the vaccine will stop the infection & $\begin{array}{l}939 \\
(24.47 \%)\end{array}$ \\
\hline I don't need the vaccine because I do all the right things: I wash my hands and wear a mask and gloves & $\begin{array}{l}1069 \\
(27.85 \%)\end{array}$ \\
\hline I don't like needles & $\begin{array}{l}238 \\
(6.20 \%)\end{array}$ \\
\hline The COVID-19 vaccine is a conspiracy & $\begin{array}{l}590 \\
(15.37 \%)\end{array}$ \\
\hline I don't need the vaccine because I'm young and healthy & $\begin{array}{l}350 \\
(9.12 \%)\end{array}$ \\
\hline I believe in natural or traditional remedies & $\begin{array}{l}253 \\
(6.59 \%)\end{array}$ \\
\hline I think COVID-19 vaccine may not be safe & $\begin{array}{l}1549 \\
(40.36 \%)\end{array}$ \\
\hline The best way for protection is to develop immunity following COVID-19 infection & $\begin{array}{l}1077 \\
(28.06 \%)\end{array}$ \\
\hline I am against vaccination in general & $\begin{array}{l}547 \\
(14.25 \%)\end{array}$ \\
\hline I had COVID-19 already so I don't need the vaccine & $\begin{array}{l}196 \\
(5.11 \%)\end{array}$ \\
\hline Other & $\begin{array}{l}18 \\
(0.47 \%)\end{array}$ \\
\hline \multicolumn{2}{|l|}{ Options to encourage future COVID-19 vaccination: } \\
\hline If my physician recommended it to me & $\begin{array}{l}863 \\
(22.49 \%)\end{array}$ \\
\hline If it was mandatory by my Job & $\begin{array}{l}830 \\
(21.63 \%)\end{array}$ \\
\hline If it was compulsory by the government $(\mathrm{MOH})$ & $\begin{array}{l}1542 \\
(40.18 \%)\end{array}$ \\
\hline If my family or friends got vaccinated & $\begin{array}{l}433 \\
(11.28 \%)\end{array}$ \\
\hline If I know that more studies showed that the vaccine is safe and effective & $\begin{array}{l}2105 \\
(54.85 \%)\end{array}$ \\
\hline If there is a way other than injection & $\begin{array}{l}142 \\
(3.70 \%)\end{array}$ \\
\hline For family protection & $\begin{array}{l}845 \\
(22.02 \%)\end{array}$ \\
\hline I would not take it in any situation & $\begin{array}{l}676 \\
(17.61 \%)\end{array}$ \\
\hline
\end{tabular}

SA $[9,10,19]$. This mistrust could be because of the rapid development of vaccines.

Our study observed multiple positive predictive factors of COVID-19 vaccination acceptance. The most significant factor was a history of influenza vaccine uptake, which was consistent with previous studies in SA [27], the US [19], UK [29], and China [18]. However, the rate of influenza vaccination in SA is low, as reported by studies in the western and central regions $[31,32]$. Given that COVID-19 is highly infectious with high death rates, a large proportion of the population should be vaccinated. Furthermore, being a man was another positive predictive factor of COVID-19 vaccine acceptance in this study, consistent with studies conducted in SA, the US, and China $[16,17,19,20]$. In contrast, although women are central to decision making about family health, there was a sex gap in COVID19 vaccine acceptance and uptake because of physical, administrative, or information accessibility issues such as myths and misinformation about the vaccine in the 
media]33[. Further studies should explore this possibility as these data can help future vaccination campaigns focus on women. Additionally, being a young adult was another positive predictor factor, which is consistent with another study conducted in SA [19]. Young adults may be more accepting of vaccination requirements than older adults for traveling or attending large gatherings]34[. In contrast, other studies conducted in the US [17] and SA [20] showed that older adults were more willing to get vaccinated than younger adults [17]. Moreover, having a high school degree or less was a positive predictor factor in this study, similar to a study conducted in SA [19]. Lack of knowledge about the nature of the vaccine may influence people to accept the vaccine. In contrast to other studies in the US [17] and SA [20], having a college or postgraduate degree was a positive predictive factor of vaccine acceptance; thus, further studies should explore this possibility as these data can be helpful for future vaccination campaigns.

Almost half of the participants refused to receive the COVID-19 vaccine (47.6\%). Most of them stated that more research was needed to validate the vaccine's safety and effectiveness before they could accept its efficacy, similar to reports from other studies in the US and SA $[15,19]$. Consistent concerns about the COVID-19 vaccine's side effects and effectiveness have been frequently reported in previous studies [29, 30, 35]. This finding could be explained by participants' education level; that is, their level of knowledge may add to their hesitation.

Most participants displayed positive beliefs regarding different aspects of the COVID-19 vaccine. They acknowledged the importance of the vaccine, its safety, and effectiveness once available. A reassuring percentage of respondents recognized that the vaccine was vital to stopping the spread, as reported in SA $[19,20]$ and Egypt [29]. Positive beliefs are fundamental because they reflect respondents' strong demand for the vaccine. This finding also highlights that participants realize the vital role of the vaccination, which provides a good start in achieving herd immunity. However, $51.9 \%$ believed that they might get infected with COVID-19 after immunization because the vaccine causes the disease. These results are consistent with those of other studies conducted in China, SA, Egypt, the US, and the UK [15, 16, 18-20, 23, 24].

Among HCWs in our study, approximately half (48.8\%) were hesitant to receive the COVID-19 vaccine. A similar result was reported in another study in SA [19]. However, a study in China showed that most HCWs were willing to receive the COVID-19 vaccine [18]. The finding regarding HCWs' acceptance of the COVID-19 vaccine in SA is concerning because they are at high risk of being infected with COVID-19 and play an important role in influencing individuals to be vaccinated.
Most parents were willing to accept vaccination for their children, consistent with the findings of two other studies conducted in SA addressing general guardians' beliefs about childhood vaccination [36, 37]. However, internationally, most parents are reluctant to vaccinate their children against COVID-19. In England and the US, participants were more likely to accept a COVID-19 vaccine for themselves than for their children because of concerns about the vaccine's safety and effectiveness [11, 38]. These studies have proposed that parents' refusal is possibly influenced by false claims that children are naturally immune to COVID-19 or cannot contract or spread the infection $[11,38]$. Furthermore, $31.43 \%$ of parents in this study were concerned about the vaccine's side effects. This fear was also observed in the US and Italy, and it was the most common reason for avoidance of childhood vaccination $[39,40]$. Considering the novelty of COVID-19 and given that future vaccines are still being developed, these concerns are understandable. Ren et al. reported that half their respondents believed that older vaccines such as polio and varicella have less risk than the new ones, supporting this point of view [41]. When further analyzing the reasons behind parents' refusal, we found that $65.44 \%$ of the respondents were doubtful about the efficacy of the vaccine.

Although wearing a mask and maintaining a safe social distance is critical for preventing the COVID-19 spread, the Centers for Disease Control and Prevention has indicated that these measures alone will not be enough to stop the virus from spreading] 42].Combining COVID-19 vaccines with these strategies will be critical in halting the pandemic. According to our findings, participants who recognized the relevance of the measures mentioned above were more eager to acquire the vaccine. Therefore, governments and health institutions should put more effort in raising public knowledge of the efficacy of these interventions. No study has investigated the use and compliance of preventive measures such as face masks, washing hands, and social distancing during the pandemic and post-pandemic. However, future studies should explore this further as it can be helpful for future vaccination campaigns.

There are substantial published studies related to COVID-19 in SA. However, few studies investigated the willingness, beliefs, and barriers for COVID-19 vaccine acceptance in SA before the release of the vaccine. Two previous studies conducted in SA $[19,20]$ have discussed these aspects; however, the present study explores these in depth and highlights some important clinical aspects regarding vaccine acceptance, especially at such an unusual time. The vaccine acceptance rate reported among our participants $(52.4 \%)$ exceeds the acceptance rate in another similar study [19]. These differences may be 
because of the timing of data collection, with a sudden increase in reported confirmed cases and the increasing number of deaths related to the COVID-19 virus, leading to changes in the perception of severity among the population. Thus, we believe that these different factors may encourage the population to receive the vaccine sooner to return to ordinary life. Moreover, this study used a larger snowball sample of 8,056 people compared to previous studies' samples of 992 [20] and 3,101 people [19]. This provided a broader insight regarding the determinants of the population's vaccine acceptance, in addition to the reasons behind their hesitancy. Furthermore, our study investigated two interesting areas, including parents' willingness to have their children vaccinated and post-pandemic attitude, which have not been discussed in previous studies in SA and remain an active area in the research field.

We may regard these data as factors in determining the severity of such incidents, which will help the government, policymakers, and healthcare professionals effectively address the population's concerns and behaviors toward COVID-19 vaccination and clarify their reasons for COVID-19 vaccine hesitancy.

\section{Conclusion}

Our study revealed a promising result of a willingness to accept the vaccine among the population, with positive beliefs and attitudes toward COVID-19 vaccination. However, a considerable number of the population were vaccine hesitant. The findings help enlighten the healthcare professionals in SA to address the population's concerns and beliefs about vaccination in general. Thus, publicly providing information about vaccine safety and implementing health education programs is crucial for increasing the public's confidence in the vaccine. The method of sharing vaccination information, in general, can be especially significant; thus, we recommend increasing public awareness about COVID-19 vaccination in our community. It is important to develop effective healthcare strategies to improve vaccination uptake for future generations. For example, promoting accurate COVID-19 vaccination information in campaigns at mosques, malls, and schools, as well as in the media, can be highly effective. In addition, we recommend that HCWs evaluate and increase information dissemination regarding the COVID-19 vaccine to increase knowledge and improve the population's attitude and practice.

\section{Limitations}

This study had some limitations. First, we conducted our study using an online questionnaire rather than face-toface interviews owing to the curfew and social distancing restrictions during the pandemic. Therefore, some biases should be considered. Second, our study was cross-sectional in nature, limiting our ability to infer causal relationships. Third, we analyzed the willingness to accept the vaccine; however, actual vaccine uptake may be lower as our study was conducted before the COVID-19 vaccine implementation started in SA (December 18, 2020). The public's intentions and attitudes may have changed since then. Fourth, we used a non-representative sample of the Saudi population because there were variations in the number of collected responses. More responses were obtained from specific regions as compared to others. The highest response rate was noted for the Middle region as Riyadh, one of the country's largest cities. This could make individuals in the region more aware of and concerned about COVID-19-related issues. Other factors may have played a role, such as Internet access and regional variances in attitudes regarding the usage of social media sites. Moreover, highly educated individuals were overrepresented in this sample, which may have impacted the results. In addition, there were some translation ambiguities in Arabic, which may have changed the meaning of some items.

\section{Abbreviations}

COVID-19: Coronavirus disease 2019; SA: Saudi Arabia; HCW: Healthcare worker; Cl: Confidence interval.

\section{Supplementary Information}

The online version contains supplementary material available at https://doi. org/10.1186/s12875-021-01606-6.

Additional file 1 (DOCX 18 kb)

\section{Acknowledgements}

The authors thank all participants who gave their time to complete the questionnaire during COVID-19 pandemic

\section{Conflict of interest}

The authors declare no conflicts of interest

\section{Authors' contributions}

Idea development and study design: NA, SMA, AAA, AFA, AMA, FA, SA, and DA. Acquiring data: NA, SMA, AAA, AFA, AMA, FA, and YA. Data analysis: YB. Drafting the manuscript: NBA, SMA, AAA, AFA, AMA, and FSA. Final writing up and critical revision of paper: YA, DA, SSA, and YB. Submission of the manuscript: YA. All authors read and approved the final manuscript.

Funding

This study received no funding

Availability of data and materials

Data that support the findings in the current study are available from the corresponding author on reasonable request.

\section{Declarations}

Ethics approval and consent to participate

This study was performed following the ethical standards of the institutional and national research Human Ethical Committee and with the 1964 Helsinki 
Declaration and its later amendments or comparable ethical standards. Informed consent was obtained from each participant or participant's guardian after receiving approval of the study by the Institutional Research Ethics Committee in King Fahad Medical City in Riyadh, Kingdom of SA (no. IRB00010471). A written informed consent was obtained from the participants after the aims of the study were explained before the survey.

\section{Consent for publication}

Not applicable

\section{Competing interests}

None of the authors have any competing interests to declare

\section{Author details}

${ }^{1}$ College of Pharmacy, King Saud University, Riyadh, Saudi Arabia. ${ }^{2}$ College of Medicine, King Saud Bin Abdul-Aziz University for Health Sciences, Riyadh, Saudi Arabia. ${ }^{3}$ College of Medicine, Imam Abdulrahman Bin Faisal University, Dammam, Saudi Arabia. ${ }^{4}$ Biostatistics Specialist, Biostatistics Department, King Fahad Medical City, Research Services Administration, Riyadh, Saudi Arabia. ${ }^{5}$ Department of Pediatrics, College of Medicine, Pediatric Infectious Diseases and Infection Control, King Saud University, Riyadh, Saudi Arabia. ${ }^{6}$ Pediatric Infectious Diseases, Pediatric Infectious Diseases Section, King Fahad Medical City, Riyadh, Saudi Arabia. ${ }^{7}$ Pediatric Emergency Department, King Fahad Medical City, Riyadh, Saudi Arabia.

Received: 10 July 2021 Accepted: 6 December 2021

Published online: 16 December 2021

\section{References}

1. W Feng W Zong F Wang S Ju 2020 Severe acute respiratory syndrome coronavirus 2 (SARS-CoV-2): a review Mol Cancer 191100 https://doi. org/10.1186/s12943-020-01218-1

2. World Health Organization. Media statement: Knowing the risks for COVID-19. https://www.who.int/indonesia/news/detail/08-03-2020knowing-the-risk-for-covid-19. Accessed Feb 192021

3. M Barry M Al Amri ZA Memish 2020 COVID-19 in the shadows of MERSCoV in the Kingdom of Saudi Arabia J Epidemiol Glob Health 10113 https://doi.org/10.2991/jegh.k.200218.003

4. World Health Organization. Coronavirus disease (COVID-19) pandemic.https://www.who.int/emergencies/diseases/novel-coron avirus-2019

5. Weqaya Public Health Authority. Saudi Center for Disease Prevention; 2021

6. WJ Guan 2020 Clinical characteristics of coronavirus disease 2019 in China N Engl J Med 3821817081720 https://doi.org/10.1056/NEJMo a2002032

7. Anon. Why a pioneering plan to distribute COVID vaccines equitably must succeed. Nature 2021;589(7841):170. doi:https://doi.org/10.1038/ d41586-021-00044-9

8. KB Habersaat C Jackson 2019 Understanding vaccine acceptance and demand-and ways to increase them Bundesgesundheitsbl 6313239 https://doi.org/10.1007/s00103-019-03063-0

9. L Palamenghi S Barello S Boccia G Graffigna 2020 Mistrust in biomedical research and vaccine hesitancy: The forefront challenge in the battle against COVID-19 in Italy Eur J Epidemiol 358785788 https://doi.org/10. 1007/s10654-020-00675-8

10. Thunstrom L. Hesitancy towards a COVID-19 vaccine and prospects for herd immunity. 2020. https://papers.ssrn.com/sol3/papers.cfm?abstract_ id=3593098. Accessed 21 Mar 2021

11. Lima $G$, et al. Public willingness to get vaccinated against COVID-19: how Al-developed vaccines can affect acceptance. 2020. https://arxiv.org/abs/ 2006.08164 Accessed 21 Mar 2021

12. M Detoc S Bruel P Frappe B Tardy E Botelho-Nevers A Gagneux-Brunon 2020 Intention to participate in a COVID-19 vaccine clinical trial and to get vaccinated against COVID-19 in France during the pandemic Vaccine 384570027006 https://doi.org/10.1016/j.vaccine.2020.09.041

13. S Neumann-Böhme 2020 Once we have it, will we use it? A European survey on willingness to be vaccinated against COVID-19 Eur J Health Econ 217977982 https://doi.org/10.1007/s10198-020-01208-6
14. Lazarus JV, et al. A global survey of potential acceptance of a COVID-19 vaccine.Nature News. https://www.nature.com/articles/s41591-0201124-9. 2020. Accessed 21 Mar 2021. doi:https://doi.org/10.1038/ s41591-020-1124-9

15. S Kreps S Prasad JS Brownstein Y Hswen BT Garibaldi B Zhang DL Kriner 2020 Factors associated with US adults' likelihood of accepting COVID-19 vaccination JAMA Netw Open 310 e2025594 https://doi.org/10.1001/ jamanetworkopen.2020.25594

16. J Wang 2020 Acceptance of COVID-19 vaccination during the COVID-19 pandemic in China Vaccines 83482 https://doi.org/10.3390/vaccines80 30482

17. AA Malik 2020 Determinants of COVID-19 vaccine acceptance in the US EClinicalMedicine 26100495 https://doi.org/10.1016/j.eclinm.2020. 100495

18. Fu C, et al. Acceptance and preference for COVID-19 vaccination in health-care workers (HCWs). medRxiv 2020. doi:https://doi.org/10.1101/ 2020.04.09.20060103

19. Magadmi R, Kamel F. Beliefs and barriers associated with COVID-19 vaccination among the general population in Saudi Arabia. Res Sq. 2020. https://www.researchsquare.com/article/rs-48955/v1 Accessed 21 Mar 2021. doi:https://doi.org/10.21203/rs.3.rs-48955/v1

20. M Al-Mohaithef BK Padhi 2020 Determinants of COVID-19 vaccine acceptance in Saudi Arabia: a web-based national survey: JMDH J Multi Health 202016571663 https://doi.org/10.2147/JMDH.S276771

21. The General Authority for Statistics. 2021. https://www.stats.gov.sa/ar/ 952. Accessed 5 Jul 2021

22. M Fadda E Albanese LS Suggs 2020 When a COVID-19 vaccine is ready, will we all be ready for it? Int J Public Health 656711712 https://doi.org/ 10.1007/s00038-020-01404-4

23. X Xiao RM Wong 2020 Vaccine hesitancy and perceived behavioral control: a meta-analysis Vaccine 383351315138 https://doi.org/10.1016/j. vaccine.2020.04.076

24. M Sallam 2021 High rates of COVID-19 vaccine hesitancy and its association with conspiracy beliefs: A study in Jordan and Kuwait among other Arab countries Vaccines 9142 https://doi.org/10.3390/vaccines9010042

25. Abdelhafiz, AS et al. Knowledge, perceptions, and attitude of Egyptians towards the novel coronavirus disease (COVID-19). J Comm Health 2020. https://www.ncbi.nlm.nih.gov/pmc/articles/PMC7173684. Accessed 21 Mar 2021. doi:https://doi.org/10.1007/s10900-020-00827-7

26. General Authority for Statistics Kingdom of Saudi Arabia

27. AM Almotairy 2019 Association between knowledge of influenza vaccine and vaccination status among general population attending primary health care centers in Al-Madinah, Saudi Arabia J Fam Med Prim Care 8929712974 https://doi.org/10.4103/jfmpc.jfmpc_547_19.22

28. AS Alqahtani 2017 Knowledge and attitudes of Saudi populations regarding seasonal influenza vaccination J Infect Public Health 106897 900 https://doi.org/10.1016/j.jiph.2017.03.011

29. World Health Organization. WHO coronavirus (COVID-19) dashboard.https://covid19.who.int/. Accessed 21 Mar 2021

30. SM Sherman LE Smith J Sim R Amlôt M Cutts H Dasch GJ Rubin N Sevdalis 2021 COVID-19 vaccination intention in the UK: results from the COVID-19 vaccination acceptability study (CoVAccS), a nationally representative cross-sectional survey Human Vaccine Immunother 1761612 1621 https://doi.org/10.1080/21645515.2020.1846397

31. M Korani 2015 Assessment of seasonal flu immunization status among adult patients visiting al-Sharaee Primary Health Care Center in Makkah al-Mokarramah Int J Med Sci Public Health 41117 https://doi.org/10. 5455/ijmsph.2015.0000201420

32. HI Al-Khashan MA Selim AM Mishriky AA Binsaeed 2011 Meningitis and seasonal influenza vaccination coverage among military personnel in central Saudi Arabia Saudi Med J 322159165

33. Gender and COVID-19 Vaccines. (2021, May). https://reliefweb.int/sites/ reliefweb.int/files/resources/GiHA\%20Gender\%20and\%20COVID19\% 20Vaccines_140621.pdf

35. M Barry 2020 Coronavirus disease-2019 pandemic in the Kingdom of Saudi Arabia: Mitigation measures and hospital preparedness J Nat Sci Med 33155158 https://doi.org/10.4103/JNSM.JNSM_29_20

36. YM AlGoraini 2020 Confidence toward vaccination as reported by parents of children admitted to a tertiary care hospital in Riyadh, Saudi Arabia: a cross sectional study Vacunas Engl Ed 21295104 https://doi. org/10.1016/j.vacune.2020.10.008 
37. TM Alshammari 2018 Parental perceptions, attitudes and acceptance of childhood immunization in Saudi Arabia: a cross sectional study Vaccine 3612328 https://doi.org/10.1016/j.vaccine.2017.11.050

38. S Bell 2020 Parents' and guardians' views on the acceptability of a future COVID-19 vaccine: a multi-methods study in England Vaccine 38497789 7798 https://doi.org/10.1016/j.vaccine.2020.10.027

39. KE Luthy RL Beckstrand LC Callister 2010 Parental hesitation in immunizing children in Utah Public Health Nurs 2712531 https://doi.org/10. 1111/j.1525-1446.2009.00823.x

40. A Facciolà 2019 Vaccine hesitancy: an overview on parents' opinions about vaccination and possible reasons of vaccine refusal J Public Health Res 811436 https://doi.org/10.4081/jphr.2019.1436

41. J Ren 2018 The demographics of vaccine hesitancy in Shanghai, China PLoS ONE 1312 e0209117 https://doi.org/10.1371/journal.pone.0209117

42. Benefits of getting a COVID-19 vaccine [Internet]. Centers for Disease Control and Prevention. https://www.cdc.gov/coronavirus/2019-ncov/ vaccines/vaccine-benefits.html

\section{Publisher's Note}

Springer Nature remains neutral with regard to jurisdictional claims in published maps and institutional affiliations

- fast, convenient online submission

- thorough peer review by experienced researchers in your field

- rapid publication on acceptance

- support for research data, including large and complex data types

- gold Open Access which fosters wider collaboration and increased citations

- maximum visibility for your research: over $100 \mathrm{M}$ website views per year

At BMC, research is always in progress.

Learn more biomedcentral.com/submissions 